United Nations Educational Scientific and Cultural Organization

and

International Atomic Energy Agency

THE ABDUS SALAM INTERNATIONAL CENTRE FOR THEORETICAL PHYSICS

\title{
SUPERSYMMETRIC SINE ALGEBRA AND DEGENERACY OF LANDAU LEVELS
}

\section{A. Jellal}

Laboratoire de Physique Théorique, (LPT-ICAC), Faculté des Sciences, Av. Ibn Battota, B.P. 1014, Rabat, Morocco

and

The Abdus Salam International Centre for Theoretical Physics, Trieste, Italy,

\section{Daoud}

Laboratoire de Physique Théorique, (LPT-ICAC), Faculté des Sciences,

Av. Ibn Battota, B.P. 1014, Rabat, Morocco,

Département de Physique, Faculté des Sciences, Université Ibn Zohr,

B.P. 28/S, Agadir, Morocco

and

The Abdus Salam International Centre for Theoretical Physics, Trieste, Italy

and

Y. Hassouni ${ }^{1}$

Laboratoire de Physique Théorique, (LPT-ICAC), Faculté des Sciences,

Av. Ibn Battota, B.P. 1014, Rabat, Morocco

and

The Abdus Salam International Centre for Theoretical Physics, Trieste, Italy.

\begin{abstract}
Two different realizations of the supersymmetric sine algebra (SSA) are given. We show that the quantum superalgebra $s l_{q}(2 / 1)$ is derived from the SSA. We discuss the relevance of the latter result to the study of spin- $\frac{1}{2}$ Bloch electron in a constant magnetic field. The relation between the deformation parameter $q$ and the degeneracy of Landau levels is established.
\end{abstract}

\section{MIRAMARE - TRIESTF}

September 1998

\footnotetext{
${ }^{1}$ Regular Associate of the Abdus Salam ICTP.
} 


\section{Introduction}

For a long time all efforts to develop the theory of symmetry in physics were restricted to the linear case, i.e. Lie groups and Lie algebras. They are seen as symmetry algebras and groups which describe linear physical systems. However, the extensive machinary largely developed for the analysis of symmetries in physics breaks down in the non-linear domain.

One notices that the non-linear algebras, having received much attention in the last few years, are the so-called quantum groups $[1,2,3]$. They are obtained by deforming the ordinary Lie algebras and are used initially for solving the quantum Yang-Baxter equation [4, 5]. The latter, seen mathematically as a representation of the braid group, has been applied in several areas of physics: conformal field theories [6,7], quantum inverse scattering [8], description of spin chains $[9,10]$ and, more recently, intermediate statistics (anyon physics) [11] and the quantum Hall effect $[12,13]$.

We also point out that recent progress in the theory of quantum groups has led to a relationship between the concept of deformation and the degeneracy of Landau levels in the description of a charged spinless particle with mass $m$ moving on a two-dimensional vector space in a constant magnetic field [14].

The aim of this work is two-fold. On one hand, we wish to convey to the reader our view that the quantum superalgebra $s l_{q}(2 / 1)$ is not only interesting from some mathematical points of view, but that it also plays an important role in the spin- $\frac{1}{2}$ Bloch electron in a uniform magnetic field. Indeed, the dimensions of the $s l_{q}(2 / 1)$ cyclic representations will be related to the degeneracy of Landau levels. Another essential result in this paper is a construction of the supersymmetric sine algebra in an original mathematical way. We also show how to obtain the quantum superalgebra $s l_{q}(2 / 1)$ from the supersymmetric sine algebra.

The material in the present paper is organized as follows. In section 2, we realize, via two different ways, the supersymmetric sine algebra (SSA). In the first step we construct it on the extended quantum plane [15]. This construction can be seen as a differential realization, since it is based on the introduction of a consistent non-commutative geometry on the quantum superplane. We discuss in the same context the realization of the SSA by using the notion of non commutative (quantum) two-torus [16]. On the other hand, we realize the SSA by considering the $k$-fermion oscillators, which can be seen as statistical objects interpolating between bosons and fermions [17].

In section 3, we show that it is possible to express the elements of the quantum superalgebra $s l_{q}(2 / 1)$ as simple combinations of the generators of the supersym metric sine algebra (SSA). This construction may be regarded as an extension of the results found in the works $[12,13,14,18]$, where the authors introduced the embedding of the quantum algebra $s l_{q}(2)$ in the sine algebra, also called the Fletcher-Floratos-Zachos (FFZ) algebra [19].

The physical application of these mathematical results is given in section 4 . We will discuss a spin- $\frac{1}{2}$ Bloch electron in a constant uniform magnetic field. The relevance of the quantum symmetry $s l_{q}(2 / 1)$ superalgebra, in the study of this simplest quantum system, is exhibited. We also show the relationship between the degeneracy of Landau levels and the deformation parameter $q$.

Conclusions are presented in the final section.

\section{Supersymmetric sine algebra (SSA)}

\subsection{Differential realization}

In this section we realize the SSA starting from two different ways. As already mentioned in the first section, we construct it in a first step by considering a non-commutative differential 
calculus discussed in [15].

Let us start by recalling these differential calculi on the quantum plane. It is introduced by considering a differential operator $d$ which satisfies:

i) the nilpotency condition: $d^{2}=0$,

ii) $d$ satisfies the graded Leibnitz rule,

iii) the differential calculus is invariant under scale transformations of coordinate points.

In the work of [15], the authors found three different differential calculi. In this paper we consider only one of them, equivalent to the following algebraic relations:

$$
\begin{array}{ll}
x d x=p d x x, & x d y=q d y x, \\
y d x=q^{-1} d x y, & y d y=r d y y, \\
d x d y=-q d x d y, &
\end{array}
$$

with $q, p$ and $r$ arbitrary complex numbers. The variables $x$ and $y$ obey the following equality

$$
x y=q y x .
$$

It is easy to verify that the relation

$$
x^{n} y^{m}=q^{n m} y^{m} x^{n},
$$

holds for any $(n, m) \in \mathbf{N}^{2}$.

The definition of the first derivatives given by

$$
\begin{aligned}
& D_{x} f(x, y)=\frac{f(p x, y)-f(x, y)}{(p-1) x}, \\
& D_{y} f(x, y)=\frac{f(x, r y)-f(x, y)}{(r-1) y}
\end{aligned}
$$

is consistent with the differential calculi introduced above. They are known in the literature as Gauss derivatives along the directions $x$ and $y \cdot \frac{1}{x}$ and $\frac{1}{y}$ are considered, in a formal sense, as objects decreasing the power of $x$ and $y$ in the analytic function $f(x, y)$. Following the relations (Eqs.(2) and (4)), one can prove the commutation relation

$$
D_{x} D_{y}=q D_{y} D_{x} .
$$

Now we will extend this differential calculus to the supersymmetric case by adding a variable $\theta$ commuting with $x$ and $y$ and obeying the relations

$$
\begin{array}{ll}
\theta^{2}=\partial_{\theta}^{2}=0, & \partial_{\theta} \theta=1-\theta \partial_{\theta}, \\
x \theta=\theta x, & x \partial_{\theta}=\partial_{\theta} x, \\
y \theta=\theta y, & y \partial_{\theta}=\partial_{\theta} y .
\end{array}
$$

The variable $\theta$ is called a fermionic variable, $\partial_{\theta}$ is the corresponding derivative.

After this brief review concerning the differential calculi on the quantum superplane, we are able to introduce the supersymmetric sine algebra (SSA) by defining the generators

$$
\begin{aligned}
& T_{m}=\left(q^{-\frac{m_{1} m_{2}}{2}} D_{x}^{m_{1}} D_{y}^{m_{2}}\right) \otimes 1, \\
& S_{m}=\left(q^{-\frac{m_{1} m_{2}}{2}} D_{x}^{m_{1}} D_{y}^{m_{2}}\right) \otimes\left(\partial_{\theta} \theta-\theta \partial_{\theta}\right) .
\end{aligned}
$$

The symbol $\otimes$ means the tensorial product between the generators belonging to the same quantum superplane described by the relations (Eqs.(1),(2),(5),(6)).

We show, by a straightforward calculation, that $T_{n}$ and $S_{n}$ satisfy

$$
\begin{aligned}
& {\left[T_{m}, T_{n}\right]_{-}=2 i \sin \left(\frac{\pi}{k} n \times m\right) T_{m+n},} \\
& {\left[T_{m}, S_{n}\right]_{-}=2 i \sin \left(\frac{\pi}{k} n \times m\right) S_{m+n},} \\
& {\left[S_{m}, S_{n}\right]_{+}=2 \cos \left(\frac{\pi}{k} n \times m\right) T_{m+n}}
\end{aligned}
$$


where $m=\left(m_{1}, m_{2}\right), n=\left(n_{1}, n_{2}\right)$ are elements of $\mathbf{Z}^{2}, n \times m=n_{1} m_{2}-n_{2} m_{1}$ and $k$ is an integer which appears after demanding $q$ to be a root of unity $\left(q=e^{\frac{2 \pi i}{k}}\right)$. We also note that $[x, y]_{-}=x y-y x$ and $[x, y]_{+}=x y+y x$ in these relations.

Consequently, we have obtained, through the equalities (Eq.(8)), and via the differential calculus on the quantum superplane introduced above, the supersymmetric sine algebra (SSA). This differential realization is possible only when $q$ is a root of unity.

A similar realization can be obtained on the non-commutative quantum two-torus. In fact, the non-commutative two-torus is defined as an associative $\mathbf{C}^{*}$-algebra $A_{h}$ which is generated by two unitary operators $U$ and $V$ satisfying the relation:

$$
U V=q V U, \quad q=e^{i h}=e^{\frac{2 \pi i}{k}} .
$$

By considering the correspondence

$$
U \longrightarrow D_{x}, \quad V \longrightarrow D_{y},
$$

the realization of the (SSA) on the quantum non-commutative two-torus becomes obvious. Now, we describe another way to lead to the realization of the SSA. We will use the $k$-fermion formalism introduced in the work of [17]. The construction is completely different from the one discussed above.

\section{2 k-fermionic realization}

Let us first introduce the $k$-fermionic algebra $\sum_{q}$ generated by an annihilation operator $a_{q}$, a creation operator $a_{q}^{+}$and a number operator $N$. The elements $a_{q}, a_{q}^{+}$and $N$ satisfy the following relations:

$$
\begin{aligned}
& a_{q} a_{q}^{+}-q a_{q}^{+} a_{q}=1, \\
& N a_{q}-a_{q} N=-a_{q}, \\
& N a_{q}^{+}-a_{q}^{+} N=a_{q}^{+}
\end{aligned}
$$

Here and in the following the complex number $q$ is chosen to be a root of unity;

$$
q=e^{\frac{2 \pi i}{k}}
$$

where $k$ is a fixed number in $\mathrm{N} /\{0,1\}$. In this case the operator $N$ is taken to be hermitian. We note that, owing to relations (Eq.(11)) with $q$ being a root of unity, the operator $a_{q}$ (respectively $a_{q}^{+}$) is not (except for $k=2$ and $k \longrightarrow \infty$ ) the adjoint of $a_{q}^{+}$(respectively $a_{q}$ ).

From the relation (Eq.(11)), we obtain

$$
\begin{aligned}
a_{q}\left(a_{q}^{+}\right)^{l} & =[l]_{q}\left(a_{q}^{+}\right)^{l-1}+q^{l}\left(a_{q}^{+}\right)^{l} a_{q}, \\
\left(a_{q}\right)^{l} a_{q}^{+} & =[l]_{q}\left(a_{q}\right)^{l-1}+q^{l} a_{q}^{+}\left(a_{q}\right)^{l},
\end{aligned}
$$

where the symbol []$_{q}$ is defined by $[x]_{q}=\frac{1-q^{x}}{1-q}$ and $l=1,2, \ldots, k-1$. In the particular case $l=k,(\mathrm{Eq} .(13))$ can be put into the form

$$
\begin{aligned}
& a_{q}\left(a_{q}^{+}\right)^{k}=\left(a_{q}^{+}\right)^{k} a_{q}, \\
& \left(a_{q}\right)^{k} a_{q}^{+}=a_{q}^{+}\left(a_{q}\right)^{k} .
\end{aligned}
$$

In addition, one can find

$$
\begin{aligned}
& N\left(a_{q}^{+}\right)^{l}=\left(a_{q}^{+}\right)^{l}(N+l), \\
& \left(a_{q}\right)^{l} N=(N+l)\left(a_{q}\right)^{l} .
\end{aligned}
$$

We remark that the relations (Eqs.(13),(14)) become trivial if we assume

$$
\left(a_{q}^{+}\right)^{k}=\left(a_{q}\right)^{k}=0 .
$$


In this section, we shall deal with a representation of the algebra $\sum_{q}$ such that (Eq.(16)) is satisfied. We note that the algebra $\sum_{-1}$ obtained for $k=2$, corresponds to ordinary fermion operators with $\left(a_{-1}^{+}\right)^{2}=\left(a_{-1}\right)^{2}=0$ which is nothing but the relation that reflects the Pauli exclusion principle. The algebra $\sum_{q}\left(q=e^{\frac{2 \pi i}{k}}\right.$ ) corresponds to quon operators (or $k$-fermion operators) $a_{q}$ and $a_{q}^{+}$interpolating between fermion and boson operators.

We continue with the situation where the constraints (Eq.(16)) hold. In this situation, we easily obtain the $k$-dimensional representation of $\sum_{q}[20,21]$ defined through

$$
\begin{aligned}
& a_{q}\left|n>=\left([n]_{q}\right)^{\frac{1}{2}}\right| n-1>, \quad a_{q} \mid 0>=0, \\
& a_{q}^{+}\left|n>=\left(\left[n+\frac{1}{2}\right]_{q}\right)^{\frac{1}{2}}\right| n+1>, \quad a_{q}^{+} \mid k-1>=0, \\
& N|n>=n| n>.
\end{aligned}
$$

where $n=0,1, \ldots, k-1$. This representation is built on a finite-dimensional (Fock) unitary space $\mathbf{F}=\{\mid n>; n=0,1, \ldots, k-1\}$ of dimension $k$. The space $\mathbf{F}$ is of dimension 2 for the fermionic algebra $\sum_{-1}$ and infinite-dimensional for the bosonic algebra $\sum_{+1}$. $\sum_{q}$,

We now introduce two operators $U$ and $V$ defined in terms of the generators of the algebra

$$
\begin{aligned}
& U=a_{q} a_{q}^{+}-a_{q}^{+} a_{q}, \\
& V=a_{q}+\frac{\left(a_{q}^{+}\right)^{k-1}}{[k-1]_{q} !} .
\end{aligned}
$$

An elementary calculation leads to

$$
U V=q^{-1} V U
$$

The latter can be iterated to produce

$$
U^{n} V^{m}=q^{-n m} V^{m} U^{n} ; \quad(n, m) \in \mathbf{N}^{2} .
$$

Let us define the operators

$$
\begin{aligned}
& K_{n}=K_{\left(n_{1}, n_{2}\right)}=\left(q^{-\frac{n_{1} n_{2}}{2}} U^{n_{1}} V^{n_{2}}\right) \otimes 1, \\
& F_{n}=F_{\left(n_{1}, n_{2}\right)}=\left(q^{-\frac{n_{1} n_{2}}{2}} U^{n_{1}} V^{n_{2}}\right) \otimes\left(a_{-} a_{-}^{+}-a_{-}^{+} a_{-}\right),
\end{aligned}
$$

with 1 the $k \times k$ identity matrix, $a_{-}$and $a_{-}^{+}$are respectively $a_{q=-1}$ and $a_{q=-1}(k=2)$.

The operators $K_{n}$ and $F_{n}$ satisfy the following commutation relations

$$
\begin{aligned}
& {\left[K_{m}, K_{n}\right]_{-}=2 i \sin \left(\frac{\pi}{k} n \times m\right) K_{m+n},} \\
& {\left[K_{m}, F_{n}\right]_{-}=2 i \sin \left(\frac{\pi}{k} n \times m\right) F_{m+n},} \\
& {\left[F_{m}, F_{n}\right]_{+}=2 \cos \left(\frac{\pi}{k} n \times m\right) F_{m+n} .}
\end{aligned}
$$

As a conclusion, the generators $K_{n}$ and $F_{n}$ can be viewed as the generators of the supersymmetric sine algebra (SSA).

We recapitulate the main results obtained in this section. We realize the SSA in two different ways. In the first one we used the differential calculi on the supersymmetric quantum plane. This construction may be regarded as a geometric one, while in the second way we have considered one $k$-fermion and one fermion (Eq.(23)).

To close this section, we recall that the quantum algebra $s l_{q}(2)$ has been obtained from sine algebra (FFZ) $[12,13,14,17,18]$. So, it is natural to think about some quantum enveloping superalgebra which can be derived from the (SSA). This idea will be treated in the following section. 


\section{The embedding of $s l_{q}(2 / 1)$ in the SSA}

This section is devoted to the construction of the quantum superalgebra $s l_{q}(2 / 1)$ from the supersymmetric sine algebra (SSA). The $s l_{q}(2 / 1)$ generators are obtained as linear combinations of those generating the SSA.

Before starting, we recall that the deformed superalgebra $s l_{q}(2 / 1)$ is generated by the set of generators $\left\{\boldsymbol{e}_{i}, f_{i}, h_{i}, i=1,2\right\}$ satisfying the following commutation relations [22]

$$
\begin{aligned}
& q^{h_{1}} q^{h_{2}}=q^{h_{2}} q^{h_{1}}, \\
& q^{h_{i}} e_{j} q^{-h_{i}}=q^{a_{i j}} e_{j}, \\
& q^{h_{i}} f_{j} q^{-h_{i}}=q^{-a_{i j}} f_{j}, \\
& e_{1} f_{1}-f_{1} e_{1}=\frac{q^{2 h_{1}-q^{-2 h_{1}}}}{q-q^{-1}}, \\
& e_{2} f_{2}+f_{2} e_{2}=\frac{q^{2 h_{2}-q^{-2 h_{2}}}}{q-q^{-1}}, \\
& e_{1} f_{2}-f_{2} e_{1}=0, \\
& e_{2} f_{1}-f_{1} e_{2}=0, \\
& e_{1}^{2} e_{2}-\left(q+q^{-1}\right) e_{1} e_{2} e_{1}+e_{2} e_{1}^{2}=0, \\
& f_{1}^{2} f_{2}-\left(q+q^{-1}\right) f_{1} f_{2} f_{1}+f_{2} f_{1}^{2}=0, \\
& e_{2}^{2}=0=f_{2}^{2},
\end{aligned}
$$

where the Cartan matrix $\left(a_{i j}\right)$ is given by

$$
\left(a_{i j}\right)=\left(\begin{array}{cc}
2 & -1 \\
-1 & 0
\end{array}\right)
$$

To obtain the $s l_{q}(2 / 1)$ superalgebra from the (SSA), we consider the generators

$$
\begin{aligned}
& L_{n}=L_{\left(n_{1}, n_{2}\right)}=T_{\left(n_{1}, n_{2}\right)} \otimes 1, \\
& M_{n}=M_{\left(n_{1}, n_{2}\right)}=T_{\left(n_{1}, n_{2}\right)} \otimes\left(\partial_{\theta} \theta-\theta \partial_{\theta}\right),
\end{aligned}
$$

where $\theta$ and $\partial_{\theta}$ are the Grassmann variable and its derivative, respectively. For convenience, we define the product between $T_{m}$ and $T_{n}$ as follows

$$
T_{m} T_{n}=q^{-\frac{m \times n}{4}} T_{m+n}
$$

where $q$ is a root of unity (i.e. $q=e^{\frac{2 \pi i}{k}}$ ).

By a direct computation, one can show that the generators $L_{m}$ and $M_{m}$ generate the SSA. From the latter, we define the operators

$$
\begin{aligned}
& e_{1}=\frac{T_{(1,1)}+T_{(-1,1)}}{q-q^{-1}} \otimes \mathbf{1}, \\
& f_{1}=-i \frac{T_{(1,-1)}-T_{(-1,-1)}}{q-q^{-1}} \otimes \mathbf{1}, \\
& q^{h_{1}}=-i T_{(-4,0)} \otimes \mathbf{1} \\
& q^{-h_{1}}=i T_{(4,0)} \otimes \mathbf{1} \\
& q^{h_{2}}=-i T_{(-4,0)} \otimes\left(\begin{array}{cc}
q^{\frac{-1}{4}} & 0 \\
0 & q^{\frac{1}{4}}
\end{array}\right), \\
& q^{-h_{2}}=i T_{(4,0)} \otimes\left(\begin{array}{cc}
q^{\frac{1}{4}} & 0 \\
0 & q^{\frac{-1}{4}}
\end{array}\right), \\
& e_{2}=\frac{T_{(1,-1)}-T_{(-1,-1)}}{\left(q-q^{-1}\right)^{\frac{1}{2}}} \otimes \partial_{\theta}, \\
& f_{2}=\frac{T_{(-1,1)}+T_{(1,1)}}{\left(q-q^{-1}\right)^{\frac{1}{2}}} \otimes \theta
\end{aligned}
$$

which satisfy equations (23) defining the quantum superalgebra $s l_{q}(2 / 1)$. Consequently, we have presented the general construction of the quantum superalgebra from the supersymmetric sine algebra. This is one of the main results of this paper. 
We point out that the construction of the quantum superalgebra $s l_{q}(2 / 1)$ from the SSA is not only interesting from a mathematical point of view, but it also has an interesting physical application. Indeed, we will show, in what follows, that $s l_{q}(2 / 1)$ is related (through the deformation parameter $q$ ) with the spin- $\frac{1}{2}$ Bloch electron in a constant uniform magnetic field.

\section{Physical application}

In this section, we show that the supersymmetric sine algebra and thus the quantum superalgebra $s l_{q}(2 / 1)$ lead to the study of the degeneracy for a physical system describing an electron moving in a constant magnetic field. In what follows we will investigate the connection between the deformation parameter $q$ and the degeneracy. To clarify all these statements, let us introduce the Hamiltonian corresponding to a spin- $\frac{1}{2}$ Bloch electron moving in the $\left(e_{x}, e_{y}\right)$-plane under the influence of a constant uniform magnetic field $\mathbf{B}=B e_{z}$ ( $e_{z}$ is normal unit vector to plane). This Hamiltonian is

$$
H=\frac{1}{2 m}\left(\pi_{x}^{2}+\pi_{y}^{2}\right)+\frac{1}{2} \hbar \omega \sigma_{z},
$$

where $\sigma_{z}$ is the Pauli spin matrix and $\omega=\frac{e B}{m c}$. The problem under consideration is supersymmetric [23] and the eigenfunctions of the Hamiltonian are labelled, in addition to the usual bosonic quantum numbers $(n, m)_{B}$, by a fermionic quantum number $n_{F}=0,1$. The latter introduces a two-fold degeneracy of each excited state.

In equation (28), the canonical momenta are defined by:

$$
\begin{aligned}
& \pi_{x}=p_{x}-\frac{e}{c} A_{x}, \\
& \pi_{y}=p_{y}-\frac{e}{c} A_{y},
\end{aligned}
$$

where $\mathbf{A}=\left(A_{x}, A_{y}\right)$ is the potential vector and can be given by

$$
\begin{aligned}
& A_{x}=-\frac{B}{2} y+\partial_{x} \Lambda, \\
& A_{y}=\frac{B}{2} x+\partial_{y} \Lambda,
\end{aligned}
$$

$\Lambda$ is an arbitrary scalar function determining the gauge we are working in. For simplicity, we choose $\Lambda=\frac{1}{2} B x y$.

As in the case of spinless electron, we define the magnetic translation operators as follows:

$$
T_{m}=T_{\left(m_{1}, m_{2}\right)}=e^{\frac{i}{\hbar} \mathbf{R}_{m} \cdot \mathbf{K}},
$$

where $R_{m}=m_{1} \mathbf{a}_{1}+m_{2} \mathbf{a}_{2}$ is an arbitrary two-dimensional vector, $m=\left(m_{1}, m_{2}\right) \in \mathbf{Z}^{2}, \mathbf{a}_{1}$ and $\mathbf{a}_{2}$ are respectively two given vectors in directions $\mathbf{e}_{x}$ and $\mathbf{e}_{y}$. The operator $\mathbf{K}$ is given by

$$
\begin{aligned}
& K_{x}=\pi_{x}-m \omega y, \\
& K_{y}=\pi_{y}+m \omega x .
\end{aligned}
$$

The components $K_{x}$ and $K_{y}$ of $\mathbf{K}$ satisfy the following commutation relation

$$
\left[K_{x}, K_{y}\right]=i \hbar \omega m .
$$

Using the Baker-Campbell-Hausdorff formula, one gets the relation

$$
T_{m} T_{n}=q^{-\frac{m \times n}{4}} T_{m+n},
$$

where $q=e^{\frac{2 \pi i}{k}}$ and $k=-\frac{h c}{2 e B \alpha_{1} \alpha_{2}}$. The scalars $\alpha_{1}$ and $\alpha_{2}$ are defined by

$$
\mathbf{a}_{1}=\alpha_{1} \mathbf{e}_{x}, \quad \mathbf{a}_{2}=\alpha_{2} \mathbf{e}_{y} .
$$


Now, we will display the relationship between the above magnetic translation operators $T_{m}$ and the generators of the supersymmetric sine algebra. Let us introduce the operators

$$
\begin{aligned}
& T_{m}^{+}=T_{m} \otimes 1, \\
& T_{m}^{-}=T_{m} \otimes\left(\sigma_{-} \sigma_{+}-\sigma_{+} \sigma_{-}\right),
\end{aligned}
$$

where the matrices $\sigma_{+}, \sigma_{-}$and $\sigma_{z}$ are given by

$$
\sigma_{+}=\left(\begin{array}{cc}
0 & 0 \\
1 & 0
\end{array}\right), \quad \sigma_{-}=\left(\begin{array}{ll}
0 & 1 \\
0 & 0
\end{array}\right), \quad \sigma_{z}=\left(\begin{array}{cc}
1 & 0 \\
0 & -1
\end{array}\right) .
$$

The generators $T_{m}^{+}$and $T_{n}^{-}$satisfy

$$
\begin{aligned}
& {\left[T_{m}^{+}, T_{n}^{+}\right]_{-}=2 i \sin \left(\frac{\pi}{2 k} n \times m\right) T_{m+n}^{+},} \\
& {\left[T_{m}^{+}, T_{n}^{-}\right]_{-}=2 i \sin \left(\frac{\pi}{2 k} n \times m\right) T_{m+n}^{-},} \\
& {\left[T_{m}^{-}, T_{n}^{-}\right]_{+}=2 \cos \left(\frac{\pi}{2 k} n \times m\right) T_{m+n}^{-}}
\end{aligned}
$$

One notices that in the limit $q \longrightarrow 1$ we recover the supersymmetric extension of the areapreserving diffeomorphisms algebra on the two dimensional surface. As before (section 3, equation $(27)$ ), we can realize the quantum superalgebra $s l_{q}(2 / 1)$ from the SSA. This result shows the relevance of $s l_{q}(2 / 1)$ in the system under consideration. More precisely, the degeneracy of Landau levels is related to the dimension of the cyclic representation of $s l_{q}(2 / 1)$. Indeed, following the work of [24], it has been proved that the representation of this quantum superalgebra has several exotic properties for $q$, a root of unity. The representation in this case is called a cyclic representation, and it has been demonstrated that the dimension of the representation space equals $2 k$. Using this result and defining the basis vectors by the ket $\left|n, m, n_{F}\right\rangle$, where $n=0,1, \ldots, \infty$ denote the energy Landau levels, $m$ is a new quantum number labelling the different quantum states for a fixed $n$ and $n_{F}$ is related to the spin state $\left(n_{F}=0,1\right)$. The action of the $s l_{q}(2 / 1)$ generators on the states $\mid n, m, n_{F}>$ is given by:

$$
\begin{aligned}
& q^{h_{1}}\left|n, m, n_{F}>=\lambda_{1}^{2} q^{-2\left(n_{F}+2 m\right)}\right| n, m, n_{F}>\quad \\
& q^{h_{2}}\left|n, m, n_{F}>=\lambda_{2}^{2} q^{2\left(n_{F}+m\right)}\right| n, m, n_{F}>, \quad(m=0,1, \ldots, k-1) \\
& f_{1}\left|n, m, n_{F}>=\varphi q^{n_{F}}\right| n, m+1, n_{F}>, \quad(m \neq k-1) \\
& f_{1}\left|n, k-1, n_{F}>=\varphi q^{n_{F}}\right| n, 0, n_{F}> \\
& e_{1}\left|n, m, n_{F}>=\varphi^{-1} q^{-n_{F}}\left(m+\mu_{2}\right)\left(\mu_{1}+\mu_{2}-m+1-n_{F}\right)\right| n, m-1, n_{F}> \\
& (m \neq 0) \\
& e_{1}\left|n, 0, n_{F}>=\varphi^{-1} q^{-n_{F}}\left(\mu_{2}\right)\left(\mu_{1}+\mu_{2}-1-n_{F}\right)\right| n, k-1, n_{F}> \\
& f_{2}\left|n, m, n_{F}>=\left(1-n_{F}\right) q^{m-1}\left(m+\mu_{2}\right)\right| n, m-1, n_{F}+1> \\
& (k \neq 0) \\
& f_{2}\left|n, 0, n_{F}>=\left(1-n_{F}\right) \lambda_{2} q^{-1}\left(\mu_{2}\right)\right| n, k-1, n_{F}+1> \\
& e_{2}\left|n, m, n_{F}>=n_{F} \lambda_{2}^{-1} q^{-m}\right| n, m+1, n_{F}-1> \\
& (k \neq l-1) \\
& e_{2}\left|n, 0, n_{F}>=n_{F} \lambda_{2}^{-1} q\right| n, k-1, n_{F}>
\end{aligned}
$$

where $\lambda_{1}, \lambda_{2}, \mu_{1}, \mu_{2}$ and $\varphi$ are complex constants determined by the cyclic properties of the representation.

The dimension of the cyclic representation $\left\{\mid n, m, n_{F}>, m=0,1, \ldots, k-1, n_{F}=0,1\right\}$ is $2 k$ which coincides, suprisingly enough, with the degeneracy of the Landau levels. This constitutes an important physical application of the mathematical results obtained in this work. Finally, we note that in the classical limit $q \longrightarrow 1(k \longrightarrow \infty)$, the system exhibits a continuous degeneracy, which is a well known result. 


\section{Conclusion}

The main result of this work is of a mathematical nature, dealing with the realization of the supersymmetric sine algebra. As in the construction of all symmetry algebras in the study of physical systems, one proceeds, in general, through a differential calculus or an oscillator realization. For linear systems, these two methods as well as, sometimes, the correspondence between, are well understood. However, to establish this correspondence in the context of quantum algebras is much more difficult. This difficulty is related to the fact that one can find many different differential calculi to realize the same algebra. The reason is that the unicity of all the introduced differential calculi remains an open question in the literature. One can think of the same problem in terms of $q$-oscillator algebras.

Therefore, in this work we have constructed the supersymmetric sine algebra following two different ways. The first one has been based on a specifc non-commutative differential calculus and the second one involves the $k$-fermion oscillator. But also establishing the correspondence between the two remains for us an open question that will be treated in a future work. Another important result of this paper is the realization of the $s l_{q}(2 / 1)$ superalgebra from the supersymmetric sine algebra. This result generalizes one well known in the context of (in)finite dimensional Lie algebras. Indeed, it has been shown in several works that $s l_{q}(2)$ can be derived from the sine algebra.

Finally, we deal with all these tools to treat one of the simplest two-dimensional quantum mechanics systems. We have proved, in fact, that the deformation parameter $q$ of the superalgebra $s l_{q}(2 / 1)$ may be related to the degeneracy of Landau levels; the degree of degeneracy of Landau levels is $2 k$ which is nothing but the dimension of the cyclic representation space of $s l_{q}(2 / 1)$.

\section{Acknowledgments}

This work was done within the framework of the Associateship Scheme of the Abdus Salam International Centre for Theoretical Physics, Trieste, Italy. One of the authors (A.J.) would like to thank Prof. A. El Hassouni for his continuous encouragement. The authors also wish to thank Prof. M. Blau for reading the manuscript and his comments.

\section{References}

[1] Drinfeld V G 1986 Quantum Group Proc. Int. Congr. Mathematicians ed A M Gleason (Providence, RI: American Mathematical Society) p798

[2] Jimbo M 1986 Lett. Math. Phys. 11 247; 1996 Commun. Math. Phys. 102537

[3] Fadeev L D, Reshetikhin N Yu and Takhtajan L A 1987 Alg. Anal. 1178

[4] Jimbo M 1989 Braid Group, Knot Theory and Statistical Mechanics ed C N Yang and M-L Ge (Singapore: World Scientific) p 111

[5] Zhang R B, Gould M D and Bracken A J 1991 Nucl. Phys. B 354625

[6] Alvarez-Gaummé L, Gomez C and Sierra G 1990 Nucl. Phys. B 330347

[7] Pasquier V and Saleur H 1990 Nucl. Phys. B 330523

[8] Faddeev L 1984 Integrable models in (1+1)-Dimensional Quantum Field theory (Les Houches Session XXXIX) ed J-B Zuber and R Stora (Amsterdam: Elsevier) course 8 
[9] Batchelor M T, Mezincescu L, Nepomechie R I and Rittenberg V 1990 J. Phys. A: Math. Gen. 23 L141

[10] Kulish P P and Sklyanin E K 1991 J. Phys. A: Math. Gen. 23 L435

[11] Lerda A and Sciuto S 1993 Nucl. Phys. B 401 613; Daoud M and Hassouni Y 1997 Mod. Phys. Lett. A12 457

[12] Kogan I I 1994 Int. J. Mod. Phys. A 96961

[13] Sato H I 1994 Mod. Phys. Lett. A 9451

[14] Chen G H, Kuang L M and Ge M L 1996 Phys. Lett. A 213231

[15] Brzezinski T, Dabrowski H and Rembienski J 1992 J. Math. Phys. 331

[16] Connes A 1986 Non-commutative differential geometry, Institut des Hautes Etudes Scientifiques. Extrait des publications mathematiques $\mathbf{6 2}$

[17] Daoud M, Hassouni Y and Kibler M 1998 The k-fermions as objects interpolating between fermions and bosons, Symmetries in Science X, eds B Gruber and M Ramek (New York: Plenum Press); ibid 1997 On the generalized coherent states, IPNL preprint.

[18] Ho H-L 1996 J. Phys. A: Math. Gen 29 L107

[19] Fairlie D, Fletcher P and Zachos C 1990 J. Math. Phys. 311088

[20] Filippov A T, Isaev A P and Kurdikov A B 1992 Mod. Phys. Lett. A7 2129; ibid 1993 Inter. J. Mod. Phys. A8 4973

[21] Daoud M, Hassouni Y 1998 Int. J. Theor. Phys. 372021

[22] Chaichain M and Kulish P P 1990 Phys. Lett. B 23472.

[23] Dereli T and Verçin A 1993 J. Phys. A: Math. Gen. 26 6961; 1992 J. Phys. B 288109

[24] Abdesselam B, Arnaudon D and Bauer M 1997 J. Phys. A: Math. Gen. 30867 